\title{
INFLUENCE OF TOTAL INERTIA EFFECTS IN A THRUST CURVILINEAR BEARING LUBRICATED WITH NEWTONIAN LUBRICANTS
}

\author{
A. WALICKA* and P. JURCZAK \\ University of Zielona Góra, Faculty of Mechanical Engineering \\ ul. Szafrana 4, 65-516 Zielona Góra, POLAND \\ E-mail: A.Walicka@ijame.uz.zgora.pl
}

\begin{abstract}
In the paper, the flow of a Newtonian type of lubricant in the clearance of a curvilinear bearing is considered. It is assumed that the bearing walls are modelled as smooth and impermeable. In analytical considerations, full inertia of the longitudinal flow and partial inertia of the circumferential flow are taken into account. The equation of motion of the lubricant is solved by the modified method of averaged inertia. A thrust bearing and spherical bearing are considered, for which dimensionless pressure distributions and the bearing capacity are given.
\end{abstract}

Key words: inertia, modified Reynolds equation, pressure distribution, load-capacity.

\section{Introduction}

Stable quasi radial flows and time-dependent squeezing flows of Newtonian fluids are used in many technical applications, especially in the engineering and chemical engineering industry. These flows are also encountered in many lubrication systems.

Flows of Newtonian fluids in the clearance between two impermeable surfaces have been considered theoretically and experimentally. The bearing walls have been modelled as two plates, two conical or spherical surfaces [1-4]. In a general case, the bearings are modelled by two surfaces of revolution [5].

The classical theory of lubrication assumes that the flow of lubricants is laminar. Usually, the inertia effects in the equations of motion are neglected [6-11]. This approximation is correct only for small values of the Reynolds number. Unfortunately, many researchers apply this assumption in the bearing applications. Many experimental and theoretical studies on the high-speed bearings show that the inertia effects of longitudinal and circumferential flows can be significant $[8,9,11,12]$.

In this paper, the Newtonian model was used to describe the lubricants behaviour in thrust hydrostatic bearings with a rotating shaft. The averaged inertia method $[12,13]$ was used to obtain the modified Reynolds equation which takes into account the inertia effects of longitudinal and circumferential flows. The solution of this equation leads to the formulae for pressure distribution and load-carrying capacity.

\section{Analysis of a lubricant flow in a bearing clearance}

Let us consider a thrust bearing with a curvilinear profile of the working surfaces and rotating pin of the shaft shown in Fig.1. The lower surface is described by the functions $R(x)$ which denotes the radius of this surface; the bearing clearance thickness is given by the function $h(x)$. An intrinsic curvilinear orthogonal coordinate system $x, \vartheta, y$ linked with the lower bearing surface is also presented in Fig.1.

\footnotetext{
* To whom correspondence should be addressed
} 


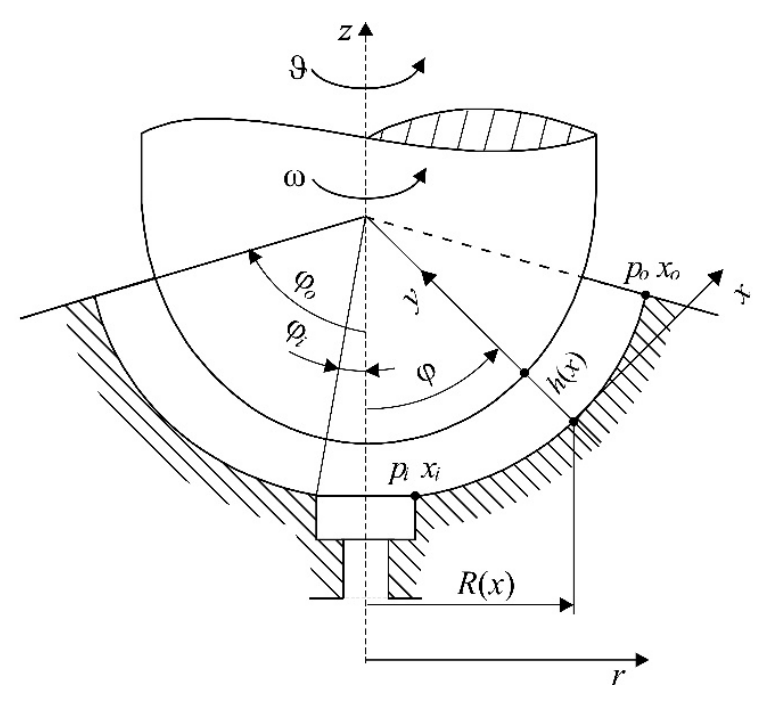

Fig.1. Configuration of the thrust hydrostatic bearing.

Taking into account the considerations of the works [11-13] we can present the equations of a Newtonian lubricant motion for axial symmetry as follows

$$
\begin{aligned}
& \frac{1}{R} \frac{\partial\left(R v_{x}\right)}{\partial x}+\frac{\partial v_{y}}{\partial y}=0 \\
& \rho\left[\frac{1}{R} \frac{\partial}{\partial x}\left(R v_{x}^{2}\right)+\frac{\partial}{\partial y}\left(v_{x} v_{y}\right)-v_{\vartheta}^{2} \frac{R^{\prime}}{R}\right]+\frac{d p}{d x}=\mu \frac{\partial^{2} v_{x}}{\partial y^{2}} \\
& \frac{\partial^{2} v_{\vartheta}}{\partial y^{2}}=0
\end{aligned}
$$

here and in what follows the prime denotes derivation with respect to $x$.

The boundary conditions for the velocity components and the pressure are as follows

$$
\begin{array}{ll}
v_{x}(x, 0)=0, & v_{x}(x, h)=0, \\
v_{\vartheta}(x, 0)=0, & v_{\vartheta}(x, h)=R \omega, \\
v_{y}(x, 0)=0, & v_{y}(x, h)=0, \\
p\left(x_{i}\right)=p_{i}, & p\left(x_{o}\right)=p_{o} .
\end{array}
$$

Integrating Eq.(2.3) and determining the arbitrary constants from the boundary conditions (2.5) we get

$$
v_{\vartheta}=R \omega \frac{y}{h} .
$$


By substituting the obtained result into Eq. (2.2) and averaging the left-hand side of Eq.(2.2) across the clearance thickness, we get

$$
f(x)=\mu \frac{\partial^{2} v_{x}}{\partial y^{2}}
$$

where:

$$
f(x)=\frac{d p}{d x}-\frac{\rho R R^{\prime} \omega^{2}}{3}+\frac{\rho}{R h} \frac{\partial}{\partial x} R \int_{0}^{h} v_{x}^{2} d y .
$$

Integrating Eq.(2.9) and taking into account the boundary conditions (2.4), we obtain

$$
v_{x}=\frac{f}{2 \mu}\left(y^{2}-y h\right)
$$

Integrating the equation of continuity (2.1) and taking into account expression (2.11) and the boundary conditions (2.6), we get

$$
\frac{1}{R} \frac{\partial}{\partial x}\left(R h^{3} f\right)=0
$$

a general form of the modified Reynolds equation.

\section{Solution to the Reynolds equation}

The solution to Eq.(2.12) has the form

$$
f=\frac{C_{1}}{R h^{3}}
$$

while the pressure distribution in the bearing clearance is given by the following equation

$$
\frac{d p}{d x}=f+\frac{\rho R R^{\prime} \omega^{2}}{3}-\frac{\rho}{R h} \frac{\partial}{\partial x} R \int_{0}^{h} v_{x}^{2} d y .
$$

To solve this equation we assume that the velocity $v_{x}$ of a lubricant flow with inertia effects is approximately equal to the velocity without inertia effects $v_{x R}: v_{x} \approx v_{x R}$.

$$
\mathrm{v}_{x} \approx \mathrm{v}_{x R} .
$$

For the Reynolds approximation (without the inertia effects) we have

$$
\frac{d p_{R}}{d x}=f_{R}=\frac{C_{1 R}}{R h^{3}}
$$


and

$$
\mathrm{v}_{x R}=\frac{1}{2 \mu} \frac{d p_{R}}{d x}\left(y^{2}-y h\right) .
$$

Substituting Eq.(3.3) into the Reynolds equation (2.12), integrating and taking into account the boundary conditions (2.7), we get

$$
p_{R}=\frac{p_{o} A_{i}-p_{i} A_{o}}{A_{i}-A_{o}}+\frac{p_{i}-p_{o}}{A_{i}-A_{o}} A(x)
$$

where

$$
A(x)=\int \frac{d x}{R h^{3}}, \quad A_{i}=A\left(x_{i}\right), \quad A_{o}=A\left(x_{o}\right) .
$$

Calculating $d p_{R} / d x$ from Eq. (3.6), we find

$$
\frac{d p_{R}}{d x}=\frac{p_{i}-p_{o}}{A_{i}-A_{o}} \frac{1}{R h^{3}}, \quad C_{l R}=\frac{p_{i}-p_{o}}{A_{i}-A_{o}}
$$

and

$$
\mathrm{v}_{x R}=\frac{C_{l R}}{2 \mu R h^{3}}\left(y^{2}-y h\right) .
$$

Substituting assumption (3.3) and putting Eqs (3.1) and (3.9) into Eq.(3.2), integrating the obtained result and taking into account the boundary conditions Eqs (2.7), we get

$$
\begin{aligned}
& \bar{p}(x)=\frac{\rho \omega^{2} R^{2}}{6}-P_{R} B(x)+\frac{\left(p_{i}-\frac{\rho \omega^{2} R_{i}^{2}}{6}+P_{R} B_{i}\right)\left[A_{o}-A(x)\right]}{A_{o}-A_{i}}+ \\
& +\frac{\left(p_{o}-\frac{\rho \omega^{2} R_{o}^{2}}{6}+P_{R} B_{o}\right)\left[A_{o}-A(x)\right]}{A_{o}-A_{i}}
\end{aligned}
$$

where

$$
P_{R}=\frac{\rho}{240 \mu^{2}}\left(\frac{p_{i}-p_{o}}{A_{i}-A_{o}}\right)^{2}, \quad B(x)=\frac{1}{(R h)^{2}} .
$$

The bearing load capacity is defined by the formula 


$$
N\left(x_{i}\right)=\pi R_{i}^{2} p_{i}+2 \pi \int_{x_{i}}^{x_{o}} \bar{p} R \cos \varphi d x
$$

whereas the sense of angle $\varphi$ arises from Fig.1.

Formula (3.12) may also be presented in the following form

$$
N\left(x_{i}\right)=\pi R_{o}^{2} p_{o}-2 \pi\left[G\left(x_{o}\right)-G\left(x_{i}\right)\right]
$$

where

$$
G(x)=\int \frac{d p}{d x}\left(\int R \cos \varphi d x\right) d x
$$

here

$$
\frac{d p}{d x}=\frac{\rho \omega^{2} R R^{\prime}}{3}+2 P_{R} \frac{(R h)^{\prime}}{(R h)^{3}}+\frac{C_{I}}{R h^{3}}
$$

and

$$
C_{1}=\frac{\left(p_{i}-\frac{\rho \omega^{2} R_{i}^{2}}{6}+P_{R} B_{i}\right)-\left(p_{o}-\frac{\rho \omega^{2} R_{o}^{2}}{6}+P_{R} B_{o}\right)}{A_{o}-A_{i}} .
$$

\section{Examples of thrust bearings}

To consider examples of thrust bearings we will transform formula (3.10) and (3.13) into a dimensionless form. To this aim let us introduce the following nondimensional parameters

$$
\begin{aligned}
& \tilde{x}=\frac{x}{x_{o}}, \quad \tilde{R}=\frac{R}{R_{o}}, \quad \varepsilon=\frac{x_{i}}{x_{o}}, \quad \tilde{h}=\frac{h}{h_{o}}, \quad \tilde{p}=\frac{\bar{p}}{p_{o}}, \quad \delta=\frac{p_{i}}{p_{o}}, \\
& \tilde{A}(\tilde{x})=A(x) \frac{h_{o}^{3} R_{o}}{x_{o}}, \quad \tilde{B}(\tilde{x})=B(x) R_{o}^{2} h_{o}^{2} \quad \tilde{N}=\frac{N-\pi R_{o}^{2} p_{o}}{\pi R_{o}^{2} p_{o}} .
\end{aligned}
$$

The dimensionless pressure distribution and load-carrying capacity are given in the following forms

$$
\tilde{p}(\tilde{x})=P_{c} \tilde{R}^{2}-P_{l} \tilde{B}(\tilde{x})+\frac{\left(\delta-P_{c} \varepsilon^{2}+P_{l} \tilde{B}_{i}\right)\left[\tilde{A}(\tilde{x})-\tilde{A}_{o}\right]}{\tilde{A}_{i}-\tilde{A}_{o}}+\frac{\left(1-P_{c}+P_{l} \tilde{B}_{o}\right)\left[\tilde{A}(\tilde{x})-\tilde{A}_{i}\right]}{\tilde{A}_{i}-\tilde{A}_{o}},
$$

and

$$
\tilde{N}(\varepsilon)=2[\tilde{G}(\varepsilon)-\tilde{G}(1)]
$$


where

$$
P_{c}=\frac{\rho \omega^{2} R_{o}^{2}}{6 p_{o}}, \quad P_{l}=\frac{P_{R}}{p_{o}}=P_{l o}\left(\frac{\delta-1}{A_{i}-A_{o}}\right)^{2}
$$

here $\left(P_{c}\right)$ is the inertia effects of the circumferential flow and $\left(P_{l}\right)$ is the inertia effects of the longitudinal flow. The coefficient

$$
P_{l o}=\frac{\rho h_{o}^{4} p_{o}}{240 \mu^{2} R_{o}^{2}}
$$

is dependent on the bearing geometry and physical lubricant properties. Its practical values are contained in the interval

$$
0 \leq P_{l o} \leq 1.0
$$

while the practical values of the coefficient $P_{c}$ are contained in the interval

$$
0 \leq P_{c} \leq 1.5
$$

Note that the zero values of the above coefficients $\left(P_{c}=0, P_{l}=0\right)$ denote the lubricant flow without inertia.

Let us consider two bearings as examples, the first of them is a radial thrust bearing shown in Fig.2 and the other one is a spherical bearing shown in Fig.3.

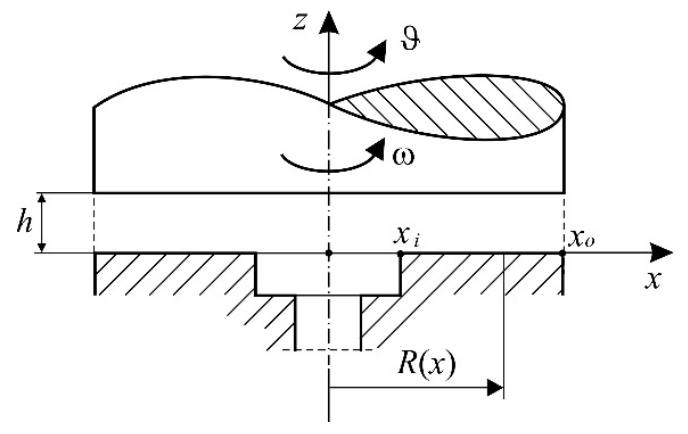

Fig.2. Configuration of the thrust plane bearing.

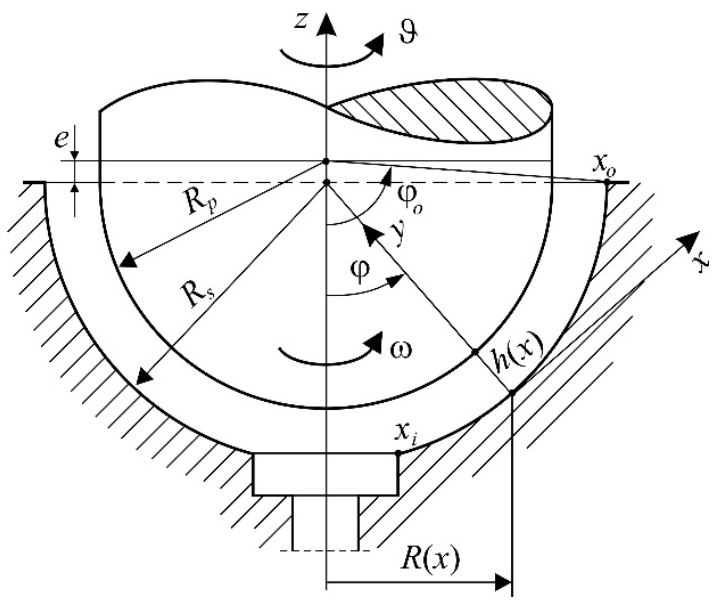

Fig.3. Configuration of the spherical bearing.

Mechanical parameters of the thrust plane bearing are described by the relationship

$$
\tilde{p}(\tilde{x})=1-P_{c}\left(1-\tilde{x}^{2}\right)+P_{l}\left(1-\frac{1}{\tilde{x}^{2}}\right)+\left[(\delta-1)+P_{c}\left(1-\varepsilon^{2}\right)-P_{l}\left(1-\frac{1}{\varepsilon^{2}}\right)\right] \frac{\ln \tilde{x}}{\ln \varepsilon},
$$

and 


$$
\tilde{N}(\varepsilon)=P_{c} \frac{\varepsilon^{4}-1}{2}+2 P_{l} \ln \varepsilon+\frac{\delta-1+P_{c}\left(1-\varepsilon^{2}\right)-P_{l}\left(1-\frac{1}{\varepsilon^{2}}\right)}{2 \ln \varepsilon}\left(\varepsilon^{2}-1\right)
$$

Figures $4 \mathrm{a}$ and $5 \mathrm{a}$ show the dimensionless pressure distribution and the load-carrying capacity for the lowspeed plane bearing, whereas Figs. $4 \mathrm{~b}$ and $5 \mathrm{~b}$ show the same parameters for the high-speed plane bearing.

a)

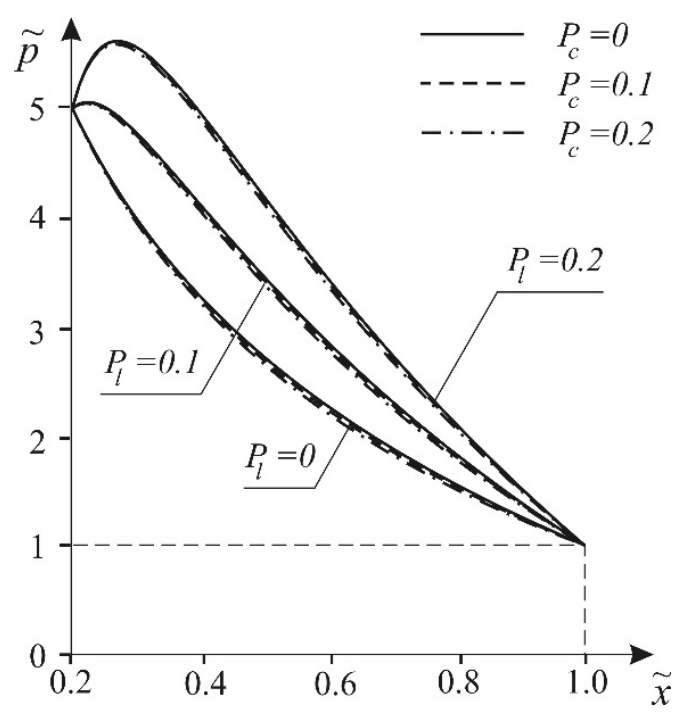

b)

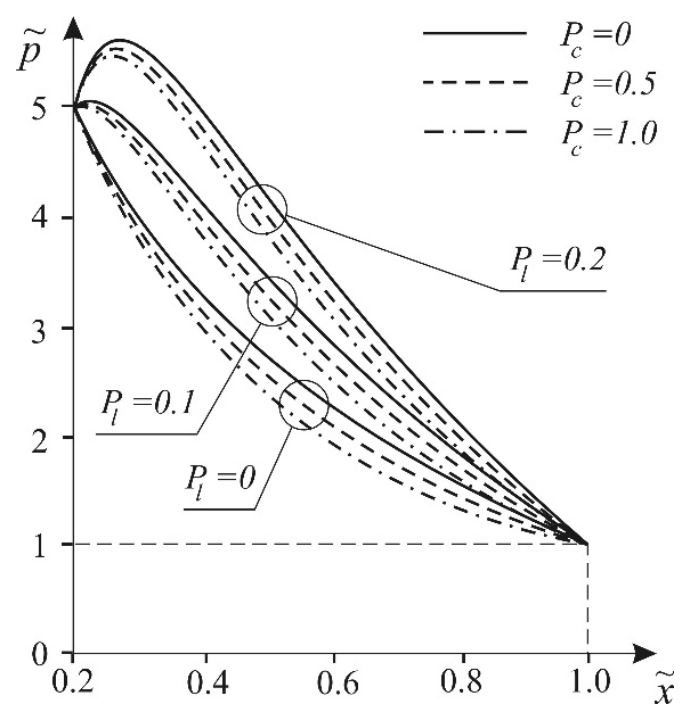

Fig.4. Dimensionless pressure distribution for the radial bearing:

a) low-speed bearing, b) high-speed bearing.

a)

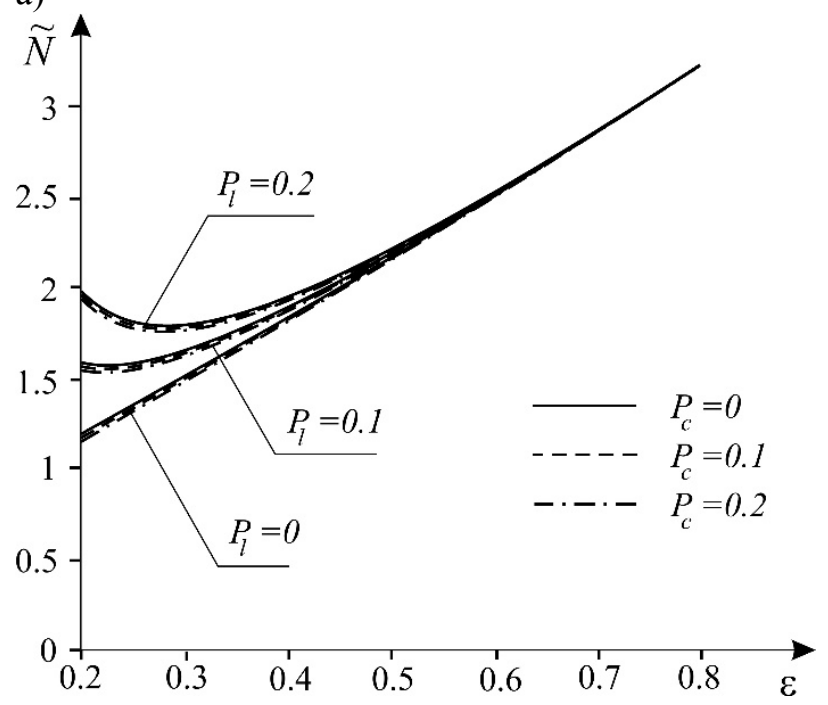

b)

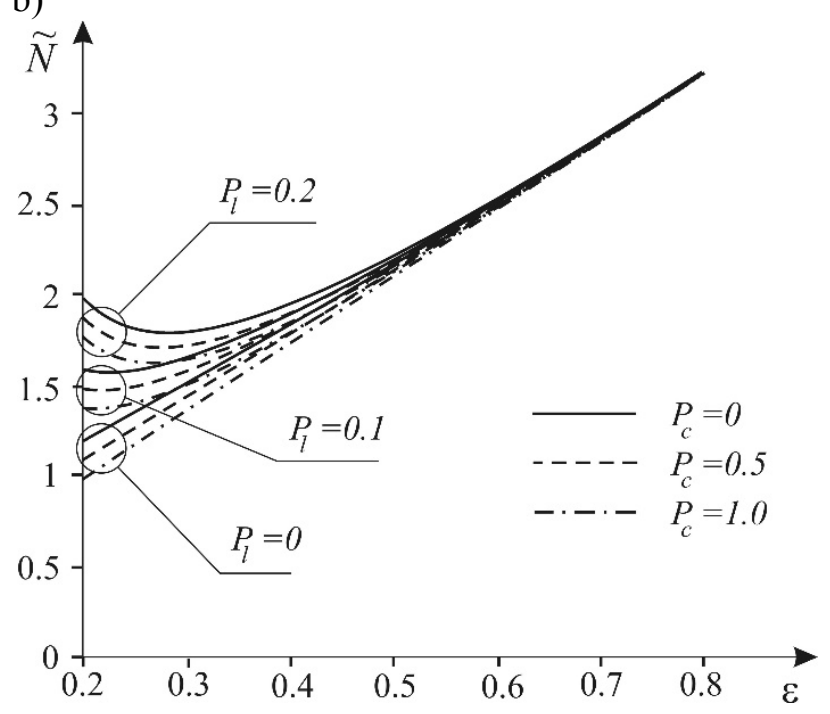

Fig.5. Dimensionless load-carrying capacity for the radial thrust bearing:

a) low-speed bearing, b) high-speed bearing.

Mechanical parameters of the spherical bearing for a clearance of constant thickness $h=h_{o}=$ const are given by the following formulae 


$$
\begin{aligned}
& \tilde{A}(\varphi)=\ln \operatorname{tg} \frac{\varphi}{2}, \quad \tilde{B}(\varphi)=\frac{1}{\sin ^{2} \varphi}, \quad P_{c}=\frac{\rho \omega^{2} R_{s}^{2}}{6 p_{o}}, \quad P_{l}=\frac{\rho h_{o}^{4} p_{o}}{240 \mu^{2} R_{s}^{2}}\left(\frac{\delta-1}{\ln \operatorname{tg} \frac{\varphi_{i}}{2}-\ln \operatorname{tg} \frac{\varphi_{o}}{2}}\right)^{2} \\
& \tilde{p}(\varphi)=P_{c} \sin ^{2} \varphi-\frac{P_{l}}{\sin ^{2} \varphi}+\left(\delta-P_{c} \sin ^{2} \varphi_{i}+\frac{P_{l}}{\sin ^{2} \varphi_{i}}\right) \frac{\ln \operatorname{tg} \frac{\varphi}{2}-\ln \operatorname{tg} \frac{\varphi_{o}}{2}}{\ln \operatorname{tg} \frac{\varphi_{i}}{2}-\ln \operatorname{tg} \frac{\varphi_{o}}{2}}+ \\
& -\left(1-P_{c} \sin ^{2} \varphi_{o}+\frac{P_{l}}{\sin ^{2} \varphi_{o}}\right) \frac{\ln \operatorname{tg} \frac{\varphi}{2}-\ln \ln \frac{\varphi_{i}}{2}}{\ln \frac{\varphi_{i}}{2}-\ln \operatorname{tg} \frac{\varphi_{o}}{2}} \\
& \tilde{N}\left(\varphi_{i}\right)=-\frac{1}{\sin ^{2} \varphi_{o}} \int_{\varphi_{i}}^{\varphi_{o}} \frac{\partial \tilde{p}}{\partial \varphi} \sin ^{2} \varphi d \varphi=\frac{1}{\sin ^{2} \varphi_{o}}\left[\tilde{G}\left(\varphi_{i}\right)-\tilde{G}\left(\varphi_{o}\right)\right]
\end{aligned}
$$

where

$$
\tilde{G}(\varphi)=\int \frac{\partial \tilde{p}}{\partial \varphi} \sin ^{2} \varphi d \varphi
$$

here

$$
\begin{aligned}
& \tilde{G}(\varphi)=\frac{P_{c} \sin ^{4} \varphi}{2}+2 P_{l} \ln \sin \varphi+ \\
& -\frac{(\delta-1)-P_{c}\left(\sin ^{2} \varphi_{i}-\sin ^{2} \varphi_{o}\right)+P_{l}\left(\frac{1}{\sin ^{2} \varphi_{i}}-\frac{1}{\sin ^{2} \varphi_{o}}\right)}{\ln \operatorname{tg} \frac{\varphi_{i}}{2}-\ln \operatorname{tg} \frac{\varphi_{o}}{2}} \cos \varphi .
\end{aligned}
$$

Therefore

$$
\begin{aligned}
& \tilde{N}\left(\varphi_{i}\right)=\frac{1}{\sin ^{2} \varphi_{o}}\left\{\frac{P_{c}}{2}\left(\sin ^{4} \varphi_{i}-\sin ^{4} \varphi_{o}\right)+2 P_{l} \ln \frac{\sin \varphi_{i}}{\sin \varphi_{o}}+\right. \\
& \left.-\left[(\delta-1)-P_{c}\left(\sin ^{2} \varphi_{i}-\sin ^{2} \varphi_{o}\right)+P_{l}\left(\frac{1}{\sin ^{2} \varphi_{i}}-\frac{1}{\sin ^{2} \varphi_{o}}\right)\right] \frac{\left(\cos \varphi_{i}-\cos \varphi_{o}\right)}{\ln \operatorname{tg} \frac{\varphi_{i}}{2}-\ln \operatorname{tg} \frac{\varphi_{o}}{2}}\right\} .
\end{aligned}
$$

Figures $6 \mathrm{a}$ and $7 \mathrm{a}$ show the dimensionless pressure distribution and the load-carrying capacity for the lowspeed spherical bearing for a clearance of constant thickness $h=h_{o}=$ const, whereas Figs $6 \mathrm{~b}$ and $7 \mathrm{~b}$ show the same parameters for the high-speed spherical bearing. 
a)

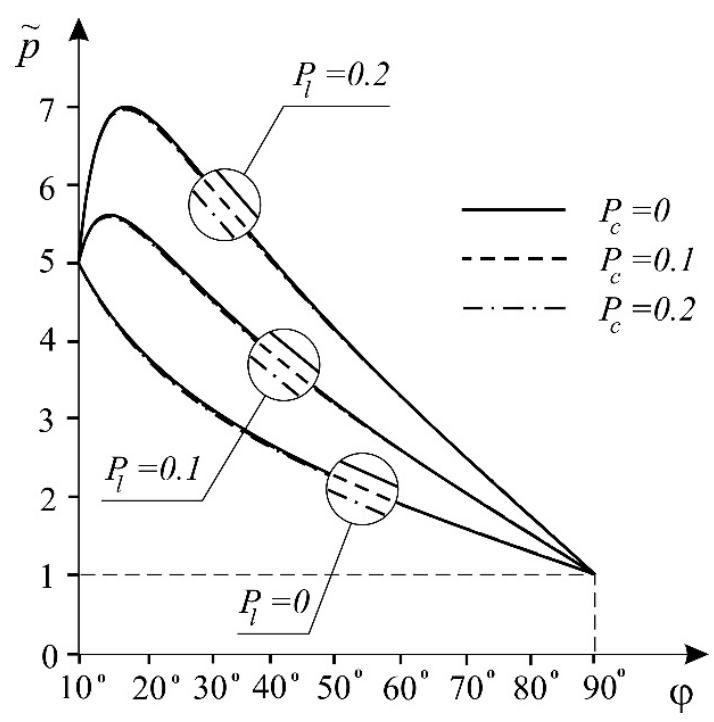

b)

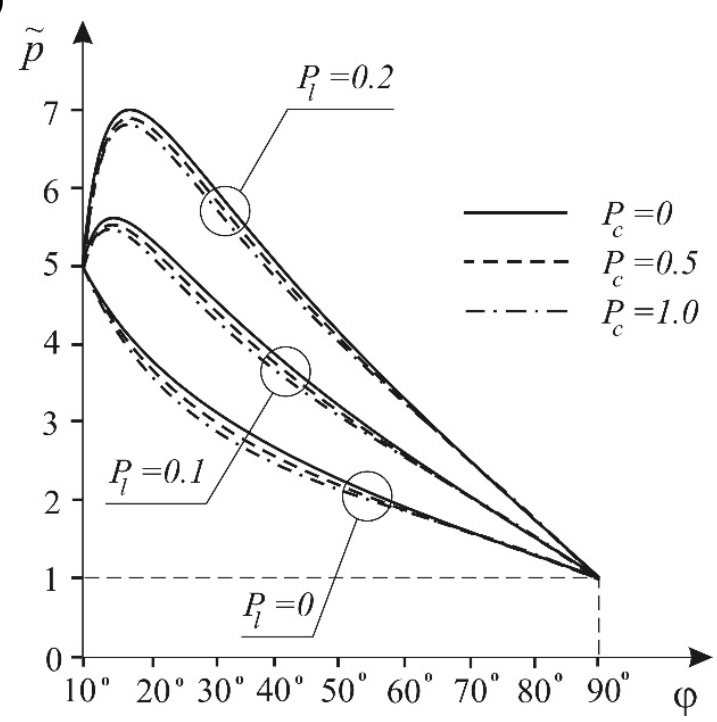

Fig.6. Dimensionless pressure distribution for the spherical bearing with a constant clearance thickness: a) low-speed bearing, b) high-speed bearing.

a)

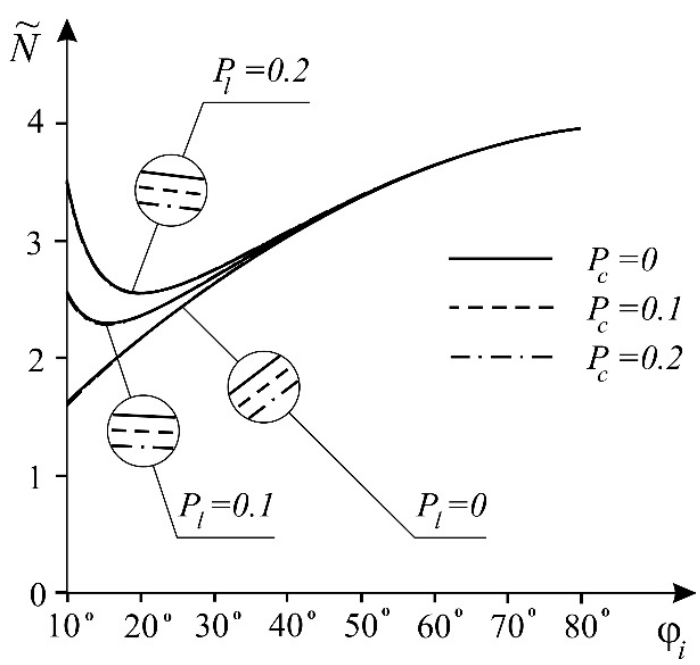

b)

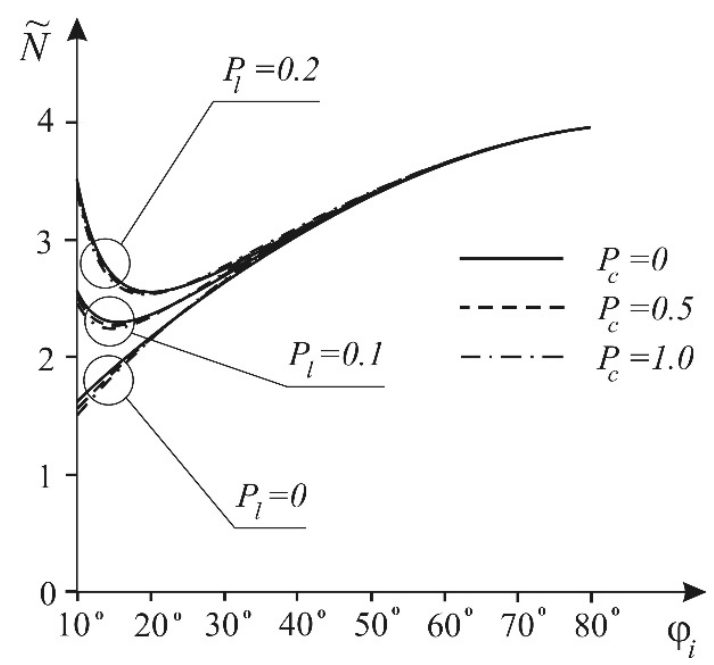

Fig.7. Dimensionless load-carrying capacity for the spherical bearing with a constant clearance thickness: a) low-speed bearing, b) high-speed bearing.

Let us consider the coincident spherical bearing $h=e \cos \varphi$ :

$$
\tilde{A}(\varphi)=\ln \operatorname{tg} \varphi+\frac{1}{2 \cos ^{2} \varphi}, \quad \tilde{B}(\varphi)=\frac{1}{\sin ^{2} \varphi \cos ^{2} \varphi}, \quad P_{c}=\frac{\rho \omega^{2} R_{s}^{2}}{6 p_{o}}, \quad P_{l}=\frac{\rho e^{4} p_{o}}{240 \mu^{2} R_{s}^{2}}\left(\frac{\delta-1}{\tilde{A}_{i}-\tilde{A}_{o}}\right)^{2},
$$


Figures 8a and 9a present the dimensionless pressure distributions and load-carrying capacity for the lowspeed spherical bearing for a clearance of the coincident bearing, whereas Figs. $8 \mathrm{~b}$ and $9 \mathrm{~b}$ show the same parameters for the high-speed spherical coincident bearing.

a)

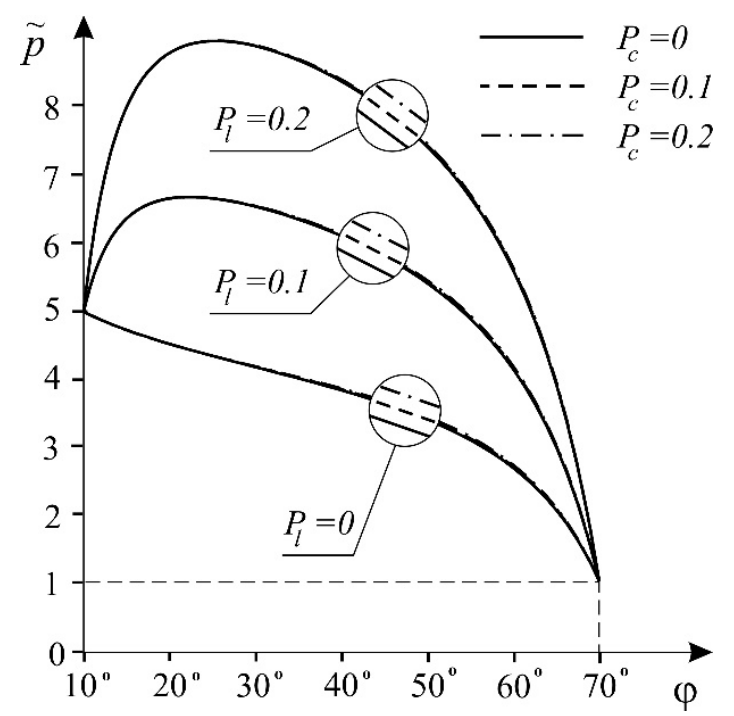

b)

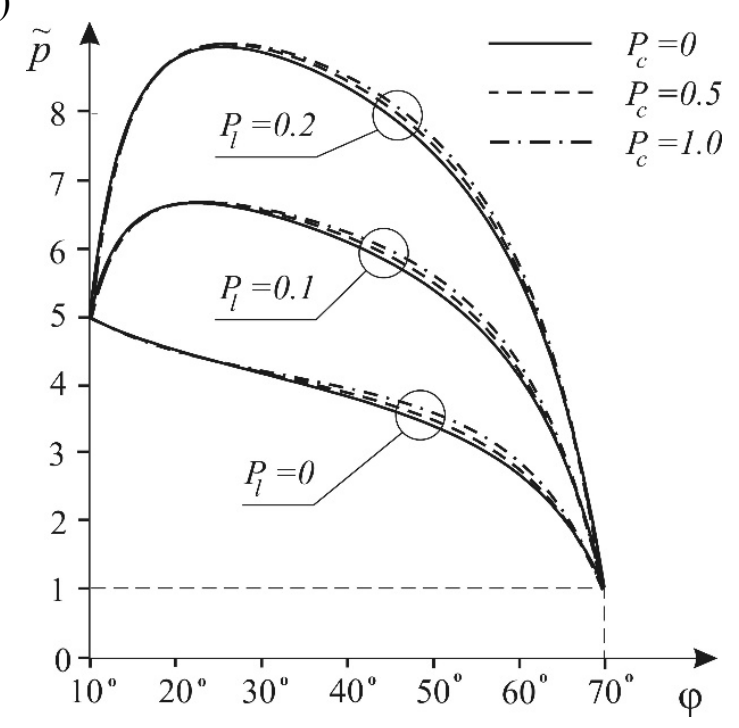

Fig.8. Dimensionless pressure distribution for the spherical coincident bearing: a) low-speed bearing, b) high-speed bearing.

a)

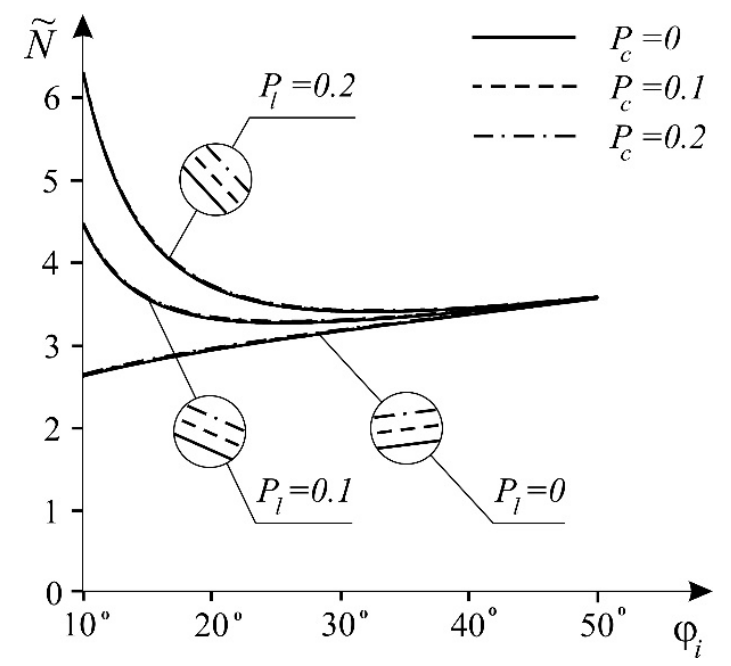

b)

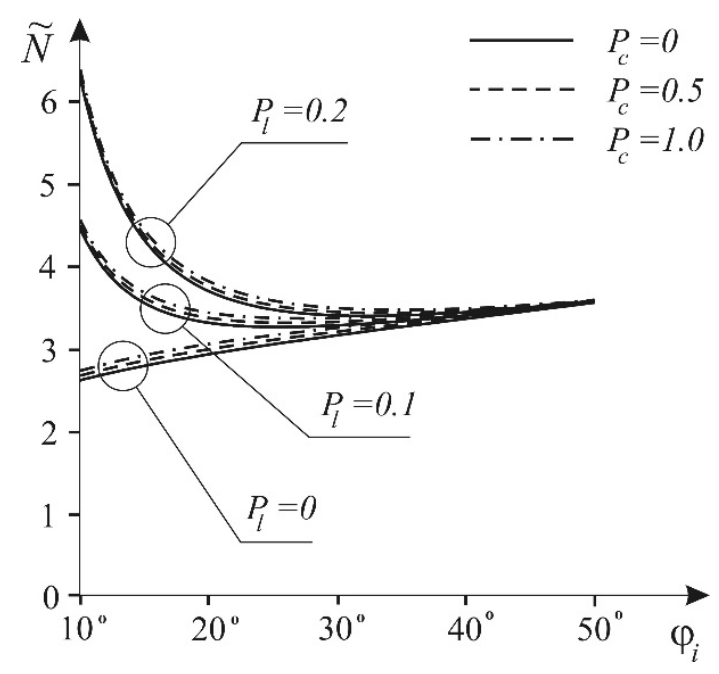

Fig.9. Dimensionless load-carrying capacity for the spherical coincident bearing: a) low-speed bearing, b) high-speed bearing.

$$
\begin{aligned}
& \tilde{p}(\varphi)=P_{c} \sin ^{2} \varphi-P_{l} \tilde{B}(\varphi)+\left(\delta-P_{c} \sin ^{2} \varphi_{i}+P_{l} \tilde{B}_{i}\right) \frac{\tilde{A}(\varphi)-\tilde{A}_{o}}{\tilde{A}_{i}-\tilde{A}_{o}}+ \\
& -\left(1-P_{c} \sin ^{2} \varphi_{o}+P_{l} \tilde{B}_{o}\right) \frac{\tilde{A}(\varphi)-\tilde{A}_{i}}{\tilde{A}_{i}-\tilde{A}_{o}}
\end{aligned}
$$




$$
\begin{aligned}
& \tilde{G}(\varphi)=\frac{P_{c} \sin ^{4} \varphi}{2}+2 P_{l}\left(\ln \operatorname{tg} \varphi-\frac{1}{2 \cos ^{2} \varphi}\right)+ \\
& +\frac{(\delta-1)-P_{c}\left(\sin ^{2} \varphi_{i}-\sin ^{2} \varphi_{o}\right)+P_{l}\left(\tilde{B}_{i}-\tilde{B}_{o}\right)}{\tilde{A}_{i}-\tilde{A}_{o}} \frac{1}{2 \cos ^{2} \varphi} .
\end{aligned}
$$

Substituting Eq.(4.16) into Eq. (4.10) we get the formula for the load-carrying capacity for the spherical coincident bearing.

For the spherical eccentricity bearing (clearance of variable thickness) $h=C(1+\varepsilon \cos \varphi)$ we have

$$
\begin{aligned}
& \tilde{A}(\varphi)=\frac{\ln (1-\cos \varphi)}{2(1+\varepsilon)^{3}}-\frac{\ln (1+\cos \varphi)}{2(1-\varepsilon)^{3}}+\frac{\varepsilon\left(3+\varepsilon^{2}\right)}{\left(1-\varepsilon^{2}\right)^{3}} \ln (1+\varepsilon \cos \varphi)+ \\
& -\frac{2 \varepsilon}{\left(1-\varepsilon^{2}\right)^{3}} \frac{1}{1+\varepsilon \cos \varphi}-\frac{\varepsilon}{2\left(1-\varepsilon^{2}\right)} \frac{1}{(1+\varepsilon \cos \varphi)^{2}}, \\
& \tilde{B}(\varphi)=\frac{1}{\sin ^{2}(1+\varepsilon \cos \varphi)^{2}}, \quad P_{c}=\frac{\rho \omega^{2} R_{s}^{2}}{6 p_{o}}, \quad P_{l}=\frac{\rho C^{4} p_{o}}{240 \mu^{2} R_{s}^{2}}\left(\frac{\delta-1}{\tilde{A}_{i}-\tilde{A}_{o}}\right)^{2},
\end{aligned}
$$

but the pressure distribution is given by formula (4.15); where

$$
\begin{aligned}
& \tilde{G}(\varphi)=\frac{P_{c} \sin ^{4} \varphi}{2}+2 P_{l}\left[\frac{\ln (1-\cos \varphi)}{2(1+\varepsilon)^{2}}+\frac{\ln (1+\cos \varphi)}{2(1-\varepsilon)^{2}}+\right. \\
& -\frac{1+\varepsilon^{2}}{\left(1-\varepsilon^{2}\right)^{2}} \ln (1+\varepsilon \cos \varphi)+\frac{1}{1-\varepsilon^{2}} \frac{1}{1+\varepsilon \cos \varphi}-\frac{1}{2(1+\varepsilon \cos \varphi)^{2}}+ \\
& \left.+\frac{(\delta-1)-P_{c}\left(\sin ^{2} \varphi_{i}-\sin ^{2} \varphi_{o}\right)+P_{l}\left(\tilde{B}_{i}-\tilde{B}_{o}\right)}{2 \varepsilon\left(\tilde{A}_{i}-\tilde{A}_{o}\right)} \frac{1}{(1+\varepsilon \cos \varphi)^{2}}\right] .
\end{aligned}
$$

and the load-carrying capacity is given by Eq.(4.10).

Figures 10a and 11a present the dimensionless pressure distributions and load-carrying capacity for a lowspeed spherical bearing for a clearance of variable thickness, $h=C(1+\varepsilon \cos \varphi)$ and Figs $10 \mathrm{~b}$ and $11 \mathrm{~b}$ show the same parameters for the high-speed spherical bearing for a clearance of variable thickness. 
a)

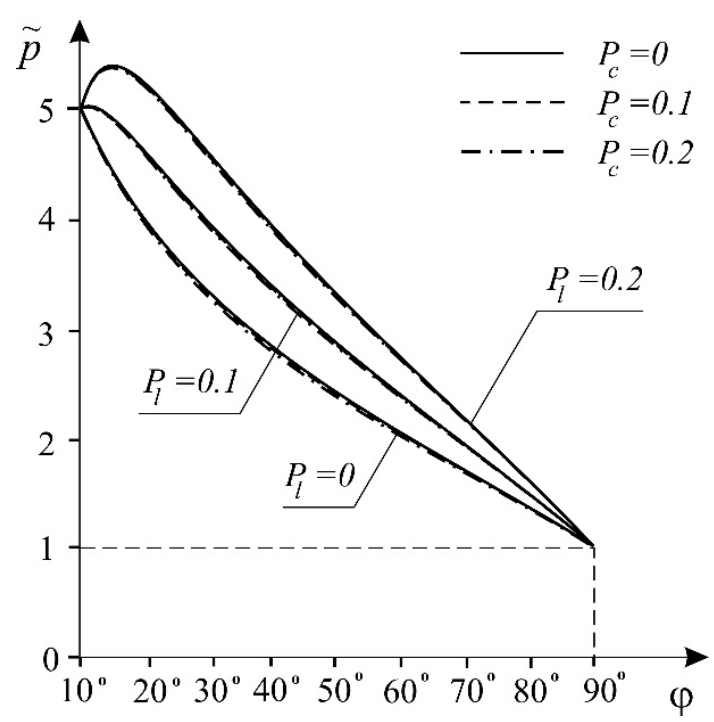

b)

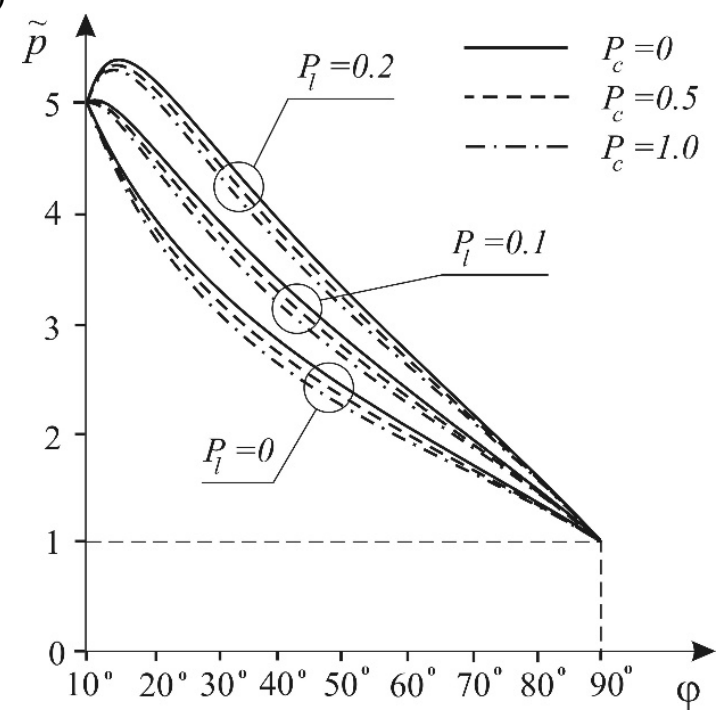

Fig.10. Dimensionless pressure distribution for the spherical bearing with a variable clearance thickness: a) low-speed bearing, b) high-speed bearing.

a)

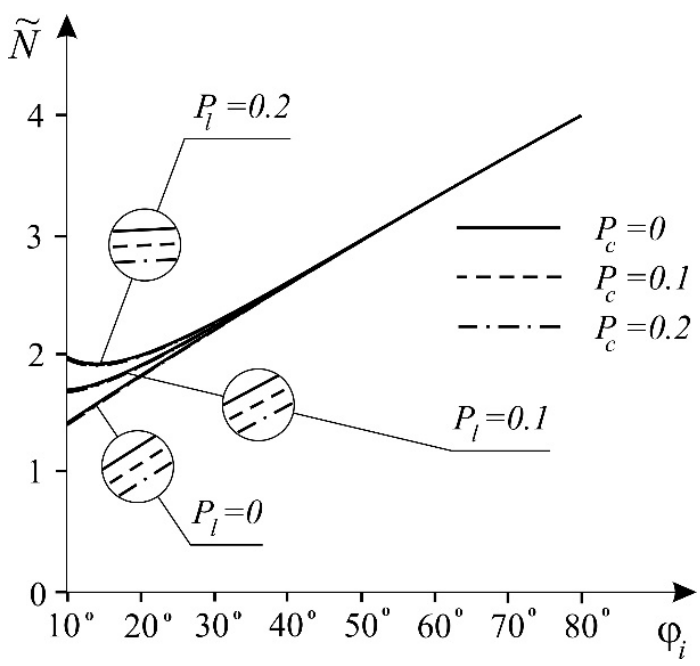

b)

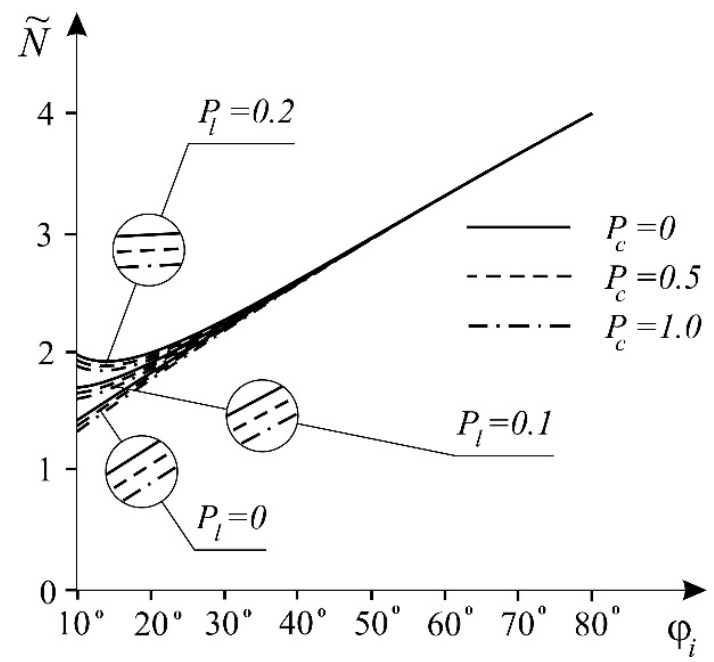

Fig.11. Dimensionless load-carrying capacity for the spherical bearing with a variable clearance thickness: a) low-speed bearing, b) high-speed bearing.

\section{Conclusion}

In this paper, a Newtonian lubricant flow in the clearance of a thrust bearing is considered. The bearing clearance is limited by two curvilinear co-working surfaces. The nondimensional pressure distributions and the load carrying capacity are calculated for the value of the parameter $\delta=5$ (the parameter $\delta=5$ determines the pressure difference between the inlet pressure $p_{i}$ and the outlet pressure $p_{o}$ ).

In the analytical considerations, full inertia of the longitudinal flow $\left(P_{l}\right)$ and partial inertia of the circumferential flow $\left(P_{c}\right)$ are considered. 
Figures 4a-11a show the mechanical parameters for low-speed bearings $\left(P_{c}=0.1 ; 0.2\right)$, while Figs $4 \mathrm{~b}-11 \mathrm{~b}$ show the mechanical parameters for high-speed bearings $\left(P_{c}=0.5 ; 1.0\right)$. For $P_{c}=0$ the bearing shaft does not rotate.

Figures 4 and 5 show the nondimensional pressure distribution and the load carrying capacity, respectively, for the thrust plane bearing. The plots show that the pressure and the load carrying capacity increases with the increase in the longitudinal inertia parameter $\left(P_{l}\right)$ compared to the plots drawn for $P_{l}=0$ (flow without the longitudinal inertia effects). With an increase of the circumferential inertia $P_{c}$ the values of pressure distributions and load-carrying capacities decrease compared to the plots drawn for $P_{c}=0$ (flow without the circumferential inertia effects). The greatest increases in the values of the mechanical parameters of the thrust plane bearing are observed near the clearance inlet (small values of $\varepsilon$ ).

Figures 6 and 7 show the dimensionless pressure distribution and the load-carrying capacity for the spherical bearing for a clearance of constant thickness $h=h_{o}=$ const . These plots show that an increase in the parameter $P_{l}$ causes essential increases in the mechanical parameters of the bearing, while an increase in the parameter $P_{c}$ causes a small decrease in the values of the mechanical parameters.

Figures 8 and 9 present the dimensionless pressure distributions and load-carrying capacity for the spherical bearing for a clearance of coincident bearing, $h=e \cos \varphi$. This plots show that the values of the mechanical parameters of the bearing increase with the increase of the values of the parameter $P_{l}$ and decrease with the increase of the values of the parameter $P_{c}$.

Figures 10 and 11 present the dimensionless pressure distributions and load-carrying capacity for the spherical bearing for a clearance of variable thickness, $h=C(1+\varepsilon \cos \varphi)$. The longitudinal inertia, expressed by $P_{l}$, results in an increase of the values of the bearing mechanical parameters, but the circumferential inertia, expressed by $P_{c}$, results in some small increase of the values of the bearing mechanical parameters.

From the analytical considerations and their graphical interpretations shown in Figs 4-11 we may conclude that the longitudinal inertia $\left(P_{l}\right)$ has a dominant influence on the increase of the values of the bearing mechanical parameters, while the circumferential inertia $\left(P_{c}\right)$ causes a small decreases of pressure and load carrying capacity. These decreases are more visible for high-speed bearings.

\section{Nomenclature}

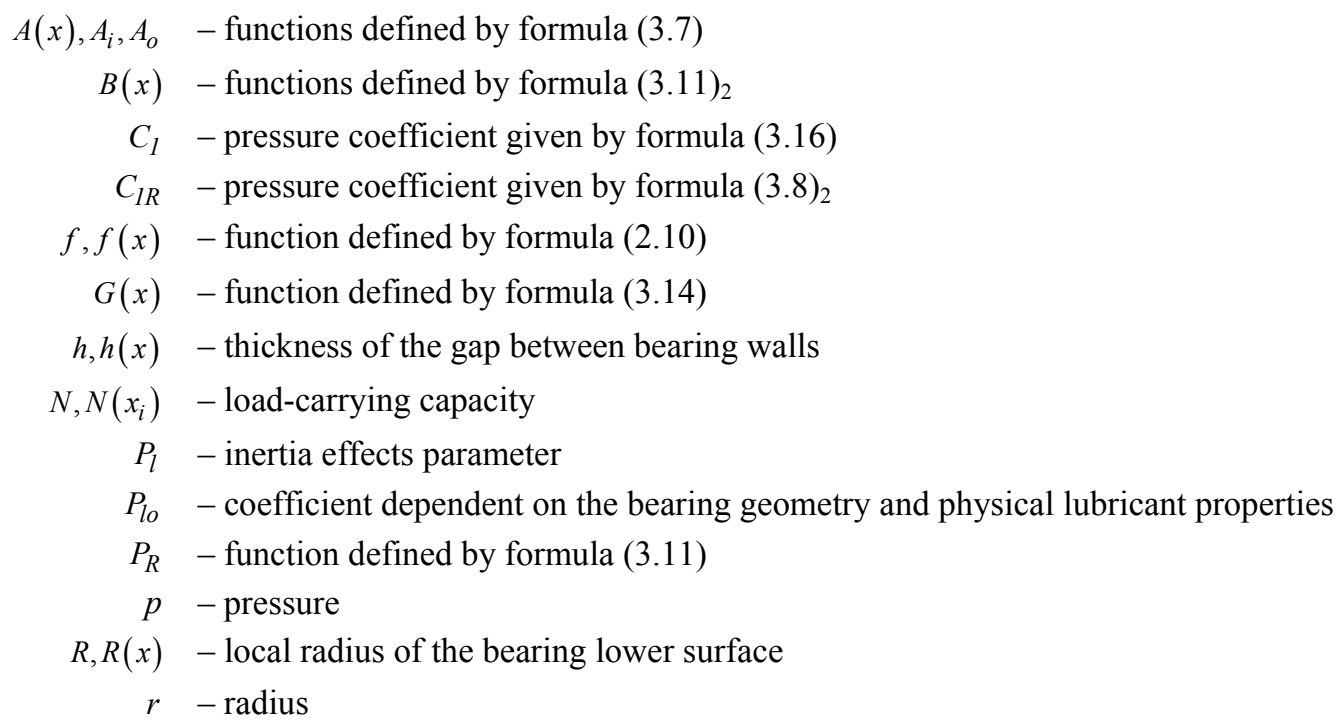


$v_{x}, v_{y}$ - velocity components

$x, \vartheta, y$ - orthogonal coordinate system

$\mu-$ viscosity coefficient

$\rho-$ density

\section{References}

[1] Agrawal V.K. (1970): Effect of lubricant inertia on squeeze film in spherical bearing. - Jap. J. Appl. Phys., vol.9, No.7, pp.831-833.

[2] Gould F. (1975): High-pressure spherical squeeze films. - J. Lubric. Technol. Trans. ASME, ser. F, vol.97, No.1, pp.207-208.

[3] Murti P.R.K. (1975): Squeeze film in curved circular plates. - J. Lubric. Technol. Trans. AME, ser F, vol.97, No.4, pp.650-654.

[4] Vora K.H. (1980): Behaviour of squeeze film between curved circular plates with a concentric circular pocket. Wear, vol.65, pp.35-38.

[5] Walicka A. (1993): Averaged inertia method for the flow of viscous fluid in a slot between fixed surfaces of revolution. - Sci.Rep. TU of Zielona Gora, no.18, pp.153-163.

[6] Möller U.J. and Boor U. (1996): Lubricants in Operations. - Düseldorf: VDI Verlag.

[7] Myshkin N.K., Kim C.K. and Petrokovets M.I. (1997): Introduction to Tribology. - Seoul, Cheong Moon Gak.

[8] Myshkin N.K. and Petrokovets M.I. (2002): Tribology. Principles and Applications (in Russian). - Gomel, IMMS NANB.

[9] Szeri A.Z. (1998): Fluid Film Lubrication. Theory and Design. - Cambridge University Press.

[10] Walicka A. and Walicki E. (2002): Surface roughness effect on the pressure distribution in curvilinear thrust bearings. - Exploitation Problems of Machines, vol.131, No.3, pp.157-167.

[11] Walicki E. (2005): Rheodynamics of Slide Bearings Lubrication (in Polish). - Zielona Góra: University Press.

[12] Walicka A. (2017): Rheology of Fluids in Mechanical Engineering. - Zielona Góra: University Press.

[13] Walicka A., Wojnarowski J. (2010): Inertia effects in a curvilinear thrust hydrostatic bearing. - Int. J. Appl. Mech. Engng, vol.15, No.3, pp.909-920.

Received: May 29, 2017

Revised: June 17, 2017 